\title{
SEQUENTIAL MOTOR ABILITY OF LEFT-HANDED INVERTED AND NON-INVERTED WRITERS
}

\author{
John I. TODOR \\ Department of Phisical Education. The University of Michigan, U.S.A.
}

Revised version received May 1979

\begin{abstract}
Left-handed female subjects, categorized as using an inverted (LI) $(n=7)$ or non-inverted ( $\mathrm{LN})(n=7)$ writing posture, were compared on performance of a motor task. Task parameters were manipulated to create four conditions varying in demand for sequential processing. Based on previously observed group differences in the direction and extent of hemispheric lateralization, non-inverted writers were predicated to exhibit superior left hand performance. An analysis of variance substantiated a performance superiority by the LN writers, however, post hoc analysis indicated the difference to exist only at the highest level of task difficulty. Analysis of right-hand performance revealed a significant group difference in favor of the LN writers and a group-by-sequential processing demand interaction. This interaction indicated that the decrement in performance of LI writers, relative to I.N writers, increased systematically with increases in task demands for sequential processing. Since previous research indicated that inverted writers exhibit less hemispheric specialization of function, it is concluded that this bilateral representation is associated with an overall reduction in sequential processing ability.
\end{abstract}

Levy and Reid $(1976,1978)$ reported the writing posture of left-handed individuals to be highly indicative of their cerebral organization. Based on the nature of the observed differences, systematic group differences in motor control should be observable. Unlike right handers, left-handed subjects are quite heterogeneous with respect to the direction of lateral specialization. It has been estimated that the cerebral organization of 50 to 70 percent of left-handers correspond to the left hemisphere/sequential or verbal processing; right hemisphere/parallel or visuospatial processing dichotomy typical of all but a few right handed subjects (Levy and Reid 1976; Milner et al. 1964; Pratt et al. 1971). In the Levy and Reid study subjects were categorized as having an inverted or non-inverted writing posture according to the relationship of the hand and pencil to the line. For left-handed non-inverted (LN) writers, the linguistic or sequential processing hemisphere was contralateral to the dominant hand, and the visuospatial or parallel processing hemisphere was ipsilateral. The reverse was true in left-handed subjects exhibiting the inverted writing posture (LI). 
In addition to the association between writing posture and the direction of hemispheric lateralization, Levy and Reid found the degree of lateralization to be related to the writing posture variable. I.I writers exhibited considerably less hemispheric specialization, i.e., relative to $L N$ writers, the hemispheric differences on either or both a verbal (sequential) and a spatial (parallel) task were less evident. According to Levy (1969), if a hemisphere is partially organized to serve both parallel and sequential functions, the performance of both will be depressed.

It is generally accepted that the distal musculature of the hands is controlled via crossed pyramidal tract pathways to and from the contralateral hemisphere (Brinkman and Kuypers 1972; Lawrence and Kuypers 1968). Additionally, the hands exhibit a performance superiority in tasks requiring the dominant processing mode of their contralateral hemisphere. For example, the right hand of right-handed subjects is superior to the left in motor tasks requiring serial organization such as rapid tapping (Lomas and Kimura 1976; Nachson and Carmon 1975; Todor and Doane 1978). Conversely, the left hand is typically superior for tactile perception (Benton et al. 1978), position reproduction (Ingram 1975; Kimura and Vanderwolf 1970; Roy and MacKenzie 1978) and ballistic movements (Todor and Doane 1978) which rely on visuospatial information and may be subserved by parallel processing.

Since Levy and Reid $(1976,1978)$ found left hand inverted writers exhibit superior sequential processing in the hemisphere ipsilateral to their dominant left hand, the efficiency of their motor control is brought to question. Unlike LN writers who have direct coupling between the hemisphere adept at serial organization and their dominant left hand, LI writers would have to use less direct mechanisms of control.

If a motor task requiring serial organization is to be performed with the left hand, LI writers may rely on one of two mechanisms: (1) transference of sensory and motor information to and from the left hemisphere via the corpus callosum; or, (2) the use of ipsilateral sensory and motor pathways. In the former, it is clear that the speed of manual response is slower when interhemispheric transfer of information is required (Berlucchi et al. 1977; Poffenberger 1912; Spirduso 1978). The significance of this increased latency would be compounded in rapid aimed movements which require repeated error correction. In the latter, in addition to having slower conduction velocities, these pathways are believed to be involved in the control of the more proximal rather than 
distal musculature of the arm (Brinkman and Kuypers 1972). Hence, if ipsilateral pathways were used, the rapid performance of precise movements would be difficult.

According to Levy and Reid, Ll writers evidence less hemispheric specialization of function. While the influence of this factor on motor performance has not been directly assessed, several indirect studies are available. Ambilaterals as a group, i.e., those with little hand difference in manual proficiency, have been found to exhibit less lateral specialization than other handedness categories (Satz et al. 1967). Several investigations using similar handedness classification techniques have found ambilaterals represent two distinct subgroups: ambilaterals exhibiting good manual dexterity with both hands who also reveal hand differences indicative of hemispheric specialization when task demands for serial or parallel processing are manipulated; and, ambilaterals with markedly inferior sequential motor performance with either hand. Unlike their high performing counterparts, the hands of low performing ambilaterals failed to show a differential hand response to manipulation of the serial and parallel processing demands of the task. On this basis it has been suggested that these ambilaterals exhibit limited hemispheric specialization of function, a factor associated with their low performance (Doane and Todor 1978; Todor and Doane 1978).

Based on the above evidence it is reasonable to predict that both the extent and direction of hemispheric specialization of LI writers should lead to inferior left hand performance of motor tasks requiring serial processing. Predictions as to the relative performance capability of the non-dominant right hand are less clear. Since the coupling between the sequential processing hemisphere and the right hand would be more direct in LI than LN writers, one would expect superior right hand manual dexterity in the LI group. However, if the consequence of less lateralized specialization is a reduction of one's general sequential processing ability, as intimated by Todor and Doane (1978), this prediction would not hold. Rather, in spite of less direct or efficient access of the LN's right hand to the sequential processing hemisphere, one would expect an LN group superiority motor performance to emerge where sequential processing demands are great.

This study contrasted the ability of left inverted and non-inverted writers to perform rapid adaptive movements with their left and right hands. 


\section{The experiment}

\section{Method}

\section{Subjects}

$S$ s were 14 college age females who write with their left hand. These $S$ s represented a subpopulation (i.e., the left-handers) of volunteers who had participated in a larger study (Todor and Kyprie 1978).

\section{Writing posture}

Ss were categorized as left-hand non-inverted (LN) $(n=7)$ or left-hand inverted (LI) $(n=7)$ writers according to two criteria: (i) whether the hand was held below (non-inverted) or above (inverted) the line of writing; and, (ii) whether the point of the pencil pointed towards the top (non-inverted) or bottom (inverted) of the page (Levy and Reid 1976). Two $S$ s could not be adequately categorized and thus were excluded from the study.

\section{Motor task}

In the context of movement control any task which requires repcated and rapid processing of error-corrective information would be expected to require sequential processing. According to Fitts (1954) the performance of rapid aimed movements is a function of the target width and the amplitude of the movement. This relationship has become known as Fitts' law in which:

\section{Index of Difficulty $(\mathrm{ID})=\log 2 \mathrm{~A} / \mathrm{W}$ bits.}

Accordingly, to increase the amplitude (A) of movement or to decrease the width (W) of the target is to increase the difficulty of a movement, i.e., the amount of error-corrective information necessary to comply with the accuracy demands of the task. Hence, given the $S$ is performing within the limits of accuracy required, the maximum speed at which controlled aimed movements can be made will be determined by the individual's information processing ability and the information required in a given condition. It is Kecle's (1973) position that the critical difference between successively greater ID's lies in the average number of error-corrective movements necessary to comply with the accuracy demands of the condition. Thus, aimed movements with a moderate to high ID would require effective sequential processing of error-corrective information if rapid performance is to be achieved.

\section{Alparatus}

A replication of Fitts (1954) reciprocal tapping task was used to assess motor ability. This apparatus consisted of two adjacent target plates bordered by error plates. In accordance with Fitts' Law, target widths $(W)$ of 0.25 .0 .50 and 1.0 inches $(0.625,1.25$ and $2.5 \mathrm{~cm}$ ) and target amplitudes (A) of 8 and 16 inches $(20.3$ and $40.6 \mathrm{~cm}$ ) were combined to produce Indices of Difficulty (ID) of 4, 5, 6, and 7 bits. The $S$ s task was to alternately tap the two adjacent targets with a hand-held stylus. The number of hits and errors per trial were independently recorded by impuise counters activated by contact of the stylus. 


\section{Procedure}

$S$ s were seated facing the apparatus with their midline centered between targets. Although instructed to alternately tap the two targets as rapidly as possible, $S$ s were cautioned that trials would be repeated if 10 percent or more of the hits were errors. Several practice movements were permitted prior to performance of each task condition. Testing started at the lowest level of difficulty (ID4 = 1.0 inch $\mathrm{W}, 8$ inch A), ascended in difficulty to ID7 (0.25 inch W, 16 inch A) and descended systematically to ID4. The hands were used alternately to perform two consecutive trials at each target width/amplitude condition, the order of hand use being counterbalanced across $S$ s.

Each trial consisted of a $5 \mathrm{sec}$ preparatory period indicated by the onset of a light, a buzzer to initiate $10 \mathrm{sec}$ of tapping which was followed by a $10 \mathrm{sec}$ rest interval. These events were automatically regulated by a Lafayette Model 52020 Programmed Timer. $S$ s were informed of both the number of correct hits and errors after each trial. The average time per movement (number of hits/10 sec) was calculated and used as the dependent measure.

\section{Results}

Fig. 1 presents the mean time per movement at the four IDs for the right and left hands of each group. The error rates were very similar between groups and did not vary systematically with ID as might be expected if speed-accuracy trade-offs were operating (range of errors (\% of total hits /trials) LN 1.5-4.6, LI 2.6-3.7).

A $2 \times 4$ (group $\times$ condition) repeated measures design analysis of variance was used to assess group differences in performance of the dominant left hand (table 1). The main effects of groups was significant indicating superior performance by the LN writers. The

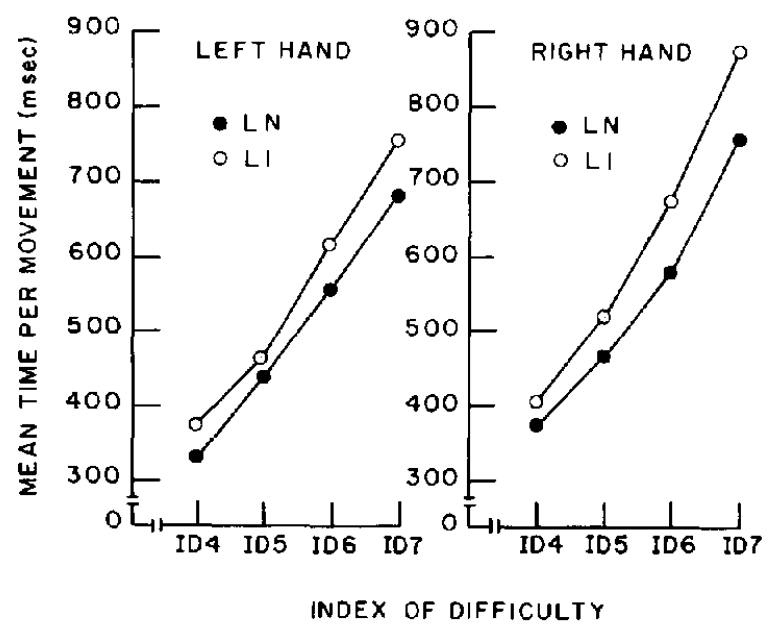

Fig. 1. Mean time per movement by ID, hand and group. 
Table 1

Analysis of variance.

\begin{tabular}{lrrr}
\hline & & & \\
Source & $d f$ & \multicolumn{1}{l}{ MS } & \\
\hline left hand & & & \\
Groups & 1 & 35.93 & 7.85 a) \\
$\quad$ Error & 12 & 4.58 & \\
Conditions & 3 & 354.23 & $467.19 \mathrm{c})$ \\
$\quad$ Error & 36 & 0.76 & \\
Condition $\times$ Group & 3 & 1.46 & 1.93 \\
$\quad$ Frror & 36 & 0.76 & \\
& & & \\
Right hand & & & \\
Groups & 1 & 74.90 & $5.50 \mathrm{a})$ \\
$\quad$ Error & 12 & 13.63 & \\
Conditions & 3 & 468.35 & $508.62 \mathrm{c})$ \\
$\quad$ Error & 36 & 0.92 & \\
Condition $\times$ Group & 3 & 4.79 & $5.20 \mathrm{~b})$ \\
$\quad$ Error & 36 & 0.92 & \\
\hline
\end{tabular}

a) $p<0.05$

b) $p<0.01$

c) $p<0.001$

main effects for condition (ID) were also significant. Furthermore, only the linear component of the performance by ID regression was significant $(F(1,12)=1082.68, p$ $<0.0001$ ), accounting for over $99 \%$ of the variability. This high degree of linearity and significant effect for condition, strongly supports Fitts' Law. The group-by-condition interaction was not statistically significant. However, $t$-tests performed at each ID indicated significant group differences to exist at the two highest levels of task difficulty (table 2). To confirm these findings and compensate for the increased probability of making a type $I$ error inherent in the use of multiple independent $t$-tests, a multivariate

Table 2

t-tests comparing groups at each ID.

\begin{tabular}{|c|c|c|c|c|}
\hline \multirow[b]{2}{*}{ ID } & \multicolumn{2}{|c|}{ Left hand } & \multicolumn{2}{|c|}{ Right hand } \\
\hline & t & $p$ & $t$ & $p$ \\
\hline ID4 & 1.85 & 0.089 & 1.24 & 0.240 \\
\hline IDS & 1.11 & 0.289 & 1.49 & 0.162 \\
\hline 11$) 6$ & 2.47 & 0.029 & 2.52 & 0.027 \\
\hline ID7 & 4.53 & 0.0007 & 3.08 & 0.0096 \\
\hline
\end{tabular}


procedure was used to derive simultaneous confidence intervals (Morrison 1976). Accordingly, the only statistically significant group difference was found at ID7 (1.99 $<72.88<143.76, p<0.05)$.

In a similar analysis for the non-dominant right hand, the main effects for groups again indicated the LN writers to be statistically superior to their LI counterparts (table 1). As with the left hand, the main effects for condition were highly significant. For the right hand, the group-by-condition interaction was significant. While the performance by ID regression of the right hand was primarily linear $(F(1,12)=2156.49, p<0.0001)$ there was a significant quadratic component $(F(1,12)=13.62, p<0.01)$. However, a group-by-condition interaction was found only for the linear component $(F(1,12)=$ $21.97, p<0.001)$. Thus, it appears the decrement in performance of the LI group, relative to $\mathrm{LN}$ writers, increased systematically with increases in ID.

\section{Discussion}

The results of the present study demonstrated the motor performance of both the left and right hands of LN writers to be superior to that of LI writers. With some qualification these differences can be accounted for by the group differences in cerebral organization described by Levy and Reid (1976, 1978).

It was predicted that as a result of either or both the direction and extent of hemispheric specialization the left hand performance of LN writers should be superior. Additionally, since the predicted differences were based on expected group differences in sequential ability, one would expect the LN writers to become increasingly superior as the task demands for sequential processing increased. While the analysis of variance substantiated the superiority of the $\mathrm{LN}$ writers' left hand performance, post hoc analysis indicated a significant difference to exist only at the highest ID. The failure to find group differences at the lower levels of task difficulty may indicate that the frequent use of the dominant left hand by LI writers can, at low levels of demand for sequential processing, compensate for any disadvantage associated with cerebral organization, i.e., less direct input/output coupling or bilateral representation.

The superior right hand performance by the $\mathrm{LN}$ writers is particularly noteworthy. Since a significant group-by-condition interaction was found, it appears that manipulation of the task demands for error-correction and thus sequential processing, amplifies performance differences in favor of the LN writers. Apparently any advantage I.I writers 
gain by the more direct coupling of the right hand and their superior sequential processing left hemisphere is overshadowed by a more generalized inferior sequential processing ability, at least as manifest in this motor task. This finding supports the contention that the bilateral representation of function characteristic of LI writers is associated with a depressed or inferior sequential processing capacity.

\section{References}

Benton, A. L., N. R. Varney, and K. de S. Hamsher, 1978. Lateral differences in tactile directional perception. Neuropsychologia 16, 109114.

Berlucchi, G., F. Crea. M. Stefano and G. Tassinari, 1977. Influence of spatial-response compatibility on reaction time of ipsilateral and contralateral hand to lateralized light stimuli. Journal of Experimental Psychology: Human Perception and Performance 3, 505-517.

Brinkman, J. and H. Kuypers, 1972. Splitbrain monkeys: cerebral control of ipsilateral and contralateral arm, hand and finger movements. Science 176, 536-539.

Doane, T. and J. I. Todor, 1978. Motor ability as a function of handedness. In: D. M. Landers and R. W. Christina (eds.), Psychology of motor behavior and sport. pp. 264- 27 I

Fitts, P.M. 1954. The Information capacity of the human motor system controlling the amplitude of movement. Journal of Experimental Psychology 47, 381391.

Ingram, D. 1975. Motor asymmetries in young children. Neuropsychologia 13, 95-10I.

Keele. S., 1973. Attention and performance. Pacific Palisades. CA.: Goodyear Publishing Co.

Kimura, D. and C. H. Vanderwolf, 1970. The relation between hand preferences and the performance of individual finger movements by left and right hands. Brain 93, 769-774.

Lawrence, D. G. and H. G. J. M. Kuypers, 1968. The functional organization of the motor system in the monkey. Brain $91,116$.

Levy, J., 1969. Possible basis for the evolution of lateral specialization of the human brain. Nature 224, 614-615.

I.cvy, J. and M. Reid, 1976. Variations in writing posture and cerebral organization. Science 194 , $337 \cdot 339$.

Levy, J. and M. Reid, 1978. Variations in cerebral organization as a function of handedness, hand posture in writing and sex. Journal of Experimental Psychology: General 107, 119-144.

Lomas, J. and D. Kimura, 1976. Intrahemispheric interaction between speaking and manual activity. Neuropsychologia 14, 2334.

Milner, B., C. Branch and T. Rasmussen 1964. Evidence for bilateral speech representation in some non-right handers. Transactions of the American Neurological Association 91, 306308.

Morrison, D. F., 1976. Multivariate statistical methods. New York: McGraw-Hill, Inc.

Nachson, I. and A. Carmon, 1975. Hand preference in sequential and spatial discrimination tasks. Cortex 11, 123.131.

Peicrs, M., 1976. Prolonged practice of a simple motor task by preferred and nonpreferred hands. Perceptual and Motor Skills 43, 447-450.

Poffenberger, A. T. 1912. Reaction time to retinal stimulation with special reference to the time lost in conduction through nerve centers. Archs Psychol. 23, 1173.

Pratt, R., E. Warrington and A. M. Halliday, 1971. Unilateral ECT as a test for cerebral dominance with a strategy for treating left handers. British Journal of Psychiatry 43, $79-83$.

Roy, E. A. and C. Mackenzie, 1978. Handedness effects in kinesthetic spatial location judyments. Cortex 14, 250 258 
Satz, P., K. Achenbach and E. Fennel, 1967. Correlations between assessed manual laterality and predicted speech laterality in a normal population. Neuropsychologia 5, 295-310.

Spirduso, W. W., 1978. Hemispheric lateralization and orientation in compensatory and voluntary movements. In: G. E. Stelmach (ed.), Information processing in motor control and learning. New York: Academic P'ress. pp. 289-309.

Todor, J. I. and T. Doane, 1978. Handedness and hemispheric asymmetry in the control of movements. Journal of Motor Behavior 10.295 300.

Todor, J. I. and P. M. Kyprie, 1978. Rapid tapping as a function of hand and handedness. Paper presented at the meetings of the Canadian Society for Psychomotor I.earning and Sports Psychology, Toronto, Ontario, Nov. 1978.

Wolff, P. H., I. Hurwite and H. Moss, 1977. Serial organization of motor skills in left-and righthanded adults. Neuropsychologia 15, 539546. 